2007-03-22

\title{
On-the-Fly Dynamic Dead Variable Analysis
}

Joel P. Self

Brigham Young University - Provo

Follow this and additional works at: https://scholarsarchive.byu.edu/etd

Part of the Computer Sciences Commons

\section{BYU ScholarsArchive Citation}

Self, Joel P., "On-the-Fly Dynamic Dead Variable Analysis" (2007). Theses and Dissertations. 886.

https://scholarsarchive.byu.edu/etd/886

This Thesis is brought to you for free and open access by BYU ScholarsArchive. It has been accepted for inclusion in Theses and Dissertations by an authorized administrator of BYU ScholarsArchive. For more information, please contact scholarsarchive@byu.edu, ellen_amatangelo@byu.edu. 


\title{
ON-THE-FLY DYNAMIC DEAD VARIABLE ANALYSIS
}

\author{
by
}

Joel Self

A thesis submitted to the faculty of Brigham Young University in partial fulfillment of the requirements for the degree of

Master of Science

Department of Computer Science

Brigham Young University

April 2007 
Copyright (c) 2007 Joel Self

All Rights Reserved 


\section{BRIGHAM YOUNG UNIVERSITY}

\section{GRADUATE COMMITTEE APPROVAL}

of a thesis submitted by

Joel Self

This thesis has been read by each member of the following graduate committee and by majority vote has been found to be satisfactory.

Date

Date

Date
Eric G. Mercer, Chair

Michael D. Jones

Eric K. Ringger 


\section{BRIGHAM YOUNG UNIVERSITY}

As chair of the candidate's graduate committee, I have read the thesis of Joel Self in its final form and have found that (1) its format, citations, and bibliographical style are consistent and acceptable and fulfill university and department style requirements; (2) its illustrative materials including figures, tables, and charts are in place; and (3) the final manuscript is satisfactory to the graduate committee and is ready for submission to the university library.

Date

Accepted for the Department

Accepted for the College
Eric G. Mercer

Chair, Graduate Committee

Parris K. Egbert

Graduate Coordinator
Thomas W. Sederberg

Associate Dean, College of Physical and Mathematical Sciences 


\title{
ABSTRACT \\ ON-THE-FLY DYNAMIC DEAD VARIABLE ANALYSIS
}

\author{
Joel Self \\ Department of Computer Science \\ Master of Science
}

State explosion in model checking continues to be the primary obstacle to widespread use of software model checking. The large input ranges of variables used in software is the main cause of state explosion. As software grows in size and complexity the problem only becomes worse. As such, model checking research into data abstraction as a way of mitigating state explosion has become more and more important. Data abstractions aim to reduce the effect of large input ranges. This work focuses on a static program analysis technique called dead variable analysis. The goal of dead variable analysis is to discover variable assignments that are not used. When applied to model checking, this allows us to ignore the entire input range of dead variables and thus reduce the size of the explored state space.

Prior research into dead variable analysis for model checking does not make full use of dynamic run-time information that is present during model checking. We present an algorithm for intraprocedural dead variable analysis that uses dynamic run-time information to find more dead variables on-the-fly and further reduce the 
size of the explored state space. We introduce a definition for the maximal state space reduction possible through an on-the-fly dead variable analysis and then show that our algorithm produces a maximal reduction in the absence of non-determinism. 


\section{ACKNOWLEDGMENTS}

I would like to thank my advisor Dr. Eric G. Mercer for his help and understanding in finishing this document. Even though I presented a less than ideal situation of trying to finish my research and thesis from a remote location while working a full-time job, he stuck with me and never became frustrated with my situation. I would also like to thank Dr. Mike D. Jones for teaching me and helping me finish my thesis. I can also confidently say that I was only able to obtain the job that I now have because of all that I learned while working for both of these gentlemen at the Software Model Checking lab.

I am also grateful to my co-workers at the lab for their help and support throughout the last 3 years. I would especially like to thank Neha Rungta for her help in getting acclimated to doing research in model checking. I also appreciate her witty updates on recent happenings at the lab. I would like to thank Joseph Edelman for being a friend and helper in the classes and work we've done together.

Additionally, I would like to thank my other labmates including Rahul Kumar, Peter Lamborn, Tonglaga Bao, and Micah Lewis.

Lastly, I would like to thank my family for their help and support throughout my life. It was only because my parents taught me the importance of education that I even considered coming this far. Most importantly I would like to thank my wife, Ashleigh, for her love and caring the whole time I worked on my Master's degree. If it were not for her I would not have made it through the toughest times. 


\section{Contents}

$0.1 \quad$ Introduction . . . . . . . . . . . . . . . . . . . . . . . . . . . . 1

$\begin{array}{lll}1 & \text { Related Work } & 6\end{array}$

2 DVA Maximal Reduction $\quad 9$

2.1 Maximal Dynamic Dead Variable Analysis . . . . . . . . . . . . . . . 12

2.2 Results . . . . . . . . . . . . . . . . . . . . . . . . . . . . . . . 18

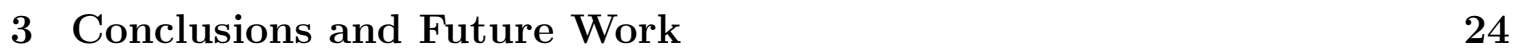




\subsection{Introduction}

Model checking is a way to automatically verify properties of a system [1, 2, 3, 4, 5]. The model of a system is a directed graph containing a set of vertices and a set of edges. In explicit state model checking, vertices represent states of the system and edges represent transitions between states. When used to verify software, model checking can discover subtle errors in deep execution traces that are easily passed over in traditional software testing techniques. Since model checking is a form of formal verification, the output of a model checker is a proof that the system does or does not satisfy the specified property.

When model checking software, a state is a snapshot of the program at a single program location. The state contains the value of the program counter and the values of all of the variables in the program. The program is used to generate successor states given a current state. Every state generated is stored in a Visited set, and every newly generated state is checked against the set to determine if the state is new. A breadth-first or depth-first search is used to explore the entire state space and ultimately verify or disprove the specified property. A single state may have multiple successors due to non-determinism in the program. Non-determinism represents input from an outside source such as user input from a keyboard or input from a sensor. The model checker must generate successor states that represent all possible input values in order to explore all possible scenarios when running the program. Since the size of the reachable state space is exponential in the branching factor of the model, the state space becomes large rather quickly, even for programs with relatively few variables. This rapid growth of the state space is called the state explosion problem.

An important technique for mitigating the state explosion problem in verification is data abstraction [6]. Data abstraction reduces the size of the generated state space by abstracting away data values; in other words, it removes variables from the state to make their value unconstrained. Variables that receive values from 
a non-deterministic input often have such large domains that removing even a single variable can greatly reduce the effect of state explosion.

Dead variable analysis is a type of data abstraction that determines when the values of variables do not matter in order to simplify a program state. Variables can be either live or dead with respect to a program location. A variable is live at a location when its current value is used. A variable is dead at a location when it is redefined before it is used in some future location, or it is not used in any future location. When a variable is dead at a program location its value does not affect the behavior of the program since it is not used. Static dead variable analysis (SDVA) has been implemented in several model checkers including SPIN, XMC, Bandera, IF, and Bebop [7, 8, 9, 10, 11]. When SDVA discovers that a variable is dead at a location, it becomes unnecessary for the model checker to track values for that variable.

We use a simple program to illustrate how SDVA helps reduce the cost of state exploration. Figure 1(a) is a simple program with labeled locations. We must assume that any possible value may be passed into the function; however, for the sake of brevity, we only consider four input patterns. The reachable state space of the program from the four input patterns is shown in Figure 1(b). There are 11 states in the state space of this program when no dead variable analysis is used. SDVA marks $c$ dead at locations $\mathbf{2}, \mathbf{3}$, and $\mathbf{4}$, since $c$ is reassigned at location $\mathbf{4}$, and it marks $b$ dead at location $\mathbf{3}$, since $b$ is reassigned at location $\mathbf{3}$. We can coalesce multiple states into a single state by ignoring dead variables since the values of these variables do not matter. For example, the states $s_{1}$ and $s_{2}$ in Figure 1(b) become equivalent when the dead variable $c$ is ignored. We combine these into one state in Figure 2(a). Similar reductions to Figure 1(b) are applied to states $\left(s_{5}, s_{6}\right),\left(s_{7}, s_{8}\right)$, and $\left(s_{10}, s_{11}\right)$. The final reduced state space from SDVA is shown in Figure 2(a).

SDVA, being a static analysis technique, does not use any of the dynamic run-time information available during model checking. For this reason, SDVA is 


\author{
1: f (int $a, \operatorname{int} b, \operatorname{int} c)$; \\ 2: if $(a>0)$ then \\ 3: $\quad b=3$; \\ 4: $c=5$ \\ 5: print a, b, c;
}

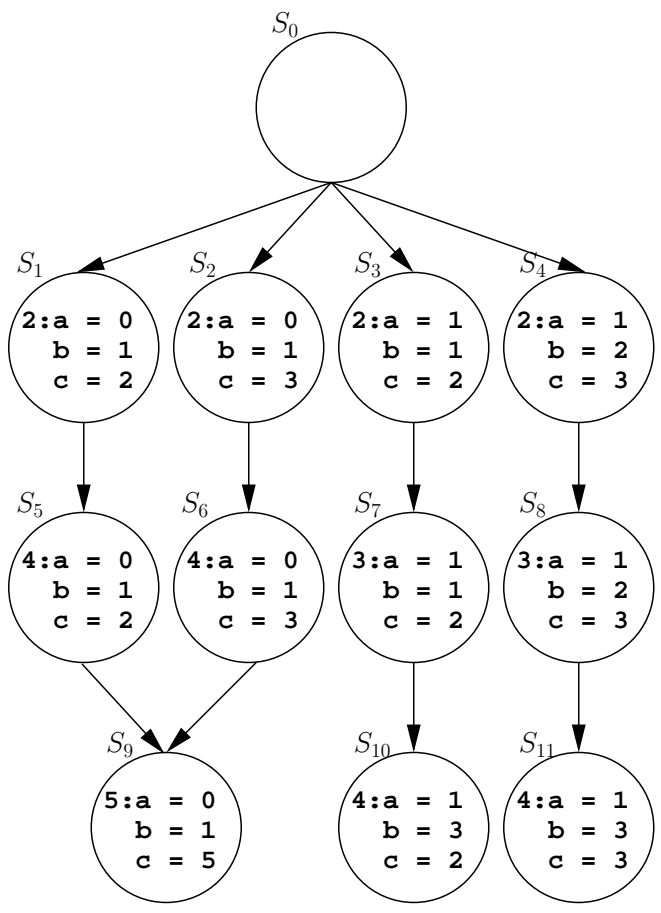

(b)

(a)

Figure 1: A small example program and state space generated from it. The program fragment in Figure 1(a) contains dead variables at various locations that can be discovered by a dead variable analysis. Figure 1(b) is a small state space generated from the program fragment.

conservative and only considers a variable dead if its current value is not used on any future paths including infeasible paths that are unreachable in any program execution. Additionally, when there is a pending pointer dereference in the program, the variable referenced cannot be known until run-time. Variable aliasing in general cannot be computed statically; therefore, in order to be safe, SDVA must assume all variables could be used at the pointer dereference and declare all variables as live. These two issues cause the SDVA to not find the true dead variable set for a state; however, run-time information that is readily available during model checking resolves memory aliasing allowing variables to be positively marked as live and other variables to be marked as dead. Run-time information also reveals the exact path taken through the program. A dead variable analysis that uses run time information during model 
checking is able to discover a more true dead variable set for each state and possibly generate smaller state spaces. This is the idea behind dynamic dead variable analysis (DDVA).

An example of the effects of DDVA can be seen in Figure 2(b). This state space is generated when variable valuations in addition to program location are used to refine dead variable analysis. When the variable $a$ is greater than zero, it causes the program to go to location $\mathbf{3}$ which makes $b$ dead at locations $\mathbf{2}$ and $\mathbf{3}$. This allows $s_{3}$ and $s_{4}$ from Figure 2(a) to be represented with just a single state, $s_{3}$, in Figure 2(b).

Recent work in DDVA labels variables live or dead dependent on specific future execution paths and is tied directly to the reachable state space of the system [12]. During model checking, [12] simulates single procedure programs forward to discover a partial future path and the variables that are referenced at pointer dereferences. The paths in the program that are not taken in the future are removed from the program. A dead variable analysis on this new program marks more variables as dead because of the missing paths; however, the DDVA algorithm requires user input to determine how far forward to simulate the program in order to achieve the greatest reduction in the state space. Without the correct input value, the algorithm achieves little to no reduction with a substantial increase in verification time and memory used. It is not possible to know what the best explore depth is a priori without further analysis. Additionally, the algorithm does not handle programs with loops and non-determinism making DDVA as implemented in [12] impractical to use.

This paper presents a definition of the maximal state space reduction possible from a dead variable analysis and a new algorithm for intraprocedural dynamic dead variable analysis that yields a maximal reduction on single procedure programs with no non-determinism. By triggering analyses only after each trace has been fully determined and by updating states in the reachable state space with new dead variable information, our new algorithm discovers the true set of dead variables for any state. 


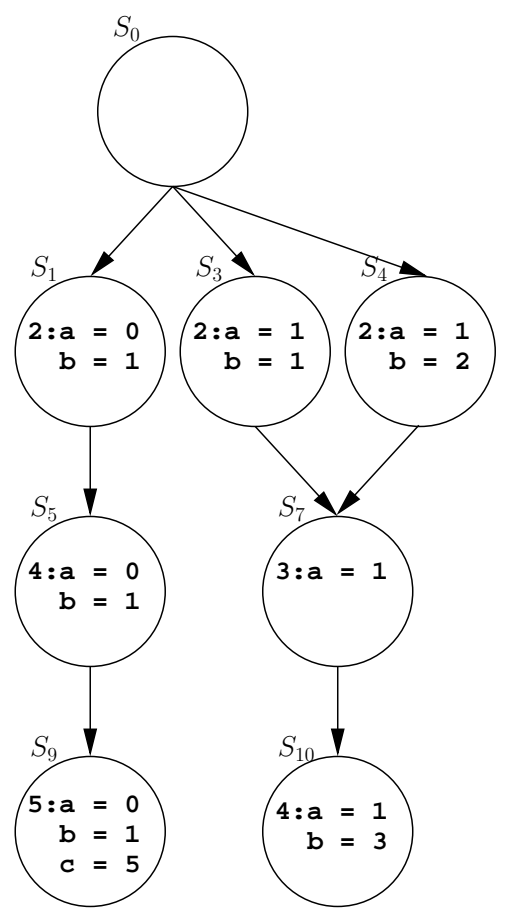

(a)

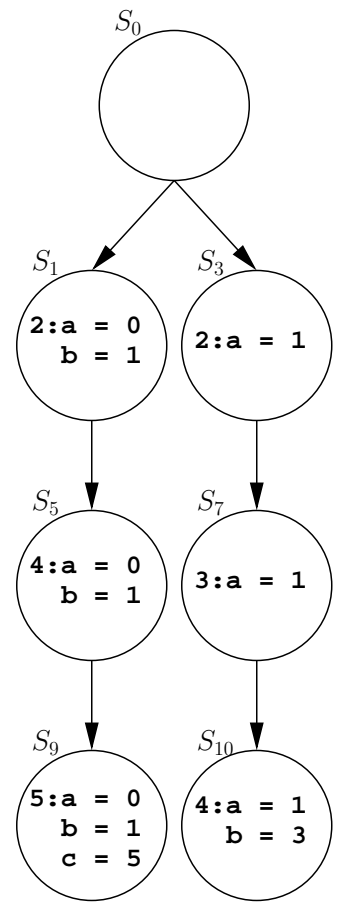

(b)

Figure 2: The effect of dead variable analysis on the state space in Figure 1(b) is illustrated in this figure. A reduction of several states from using SDVA is shown in Figure 2(a). The additional reduction of another state from using a DDVA is shown in Figure 2(b).

Without non-determinism, the future of an execution path is fixed; however, with non-determinism, the future path is uncertain. A single state can have a future that causes one of its variables be dead and another future where that same variable is live. Variables that become dead after a point of non-determinism cannot be reliably marked as dead before the point of non-determinism without first analyzing the entire reachable state space. In the presence of non-determinism, our algorithm yields the maximum state space reduction that is possible from an on-the-fly dead variable analysis. 


\section{Chapter 1}

\section{Related Work}

There are currently two relevant works on dead variable analysis in model checking known to us. The work in [10] focuses primarily on showing that live variable analysis defines an equivalence stronger than bisimulation equivalence. Static live variable analysis comes at virtually no cost compared to the cost of model checking and is completely orthogonal to other techniques used to attack the state explosion problem. Although SDVA is relatively quick, it only considers program locations in its analysis and can only discover unconditionally dead variables. An analysis that makes use of variable valuations available during model checking, in addition to program locations, can determine more precise paths through the program and find variables that are conditionally dead.

The dynamic dead variable analysis in [12] uses run time information to resolve conditional branches and pointer dereferences. In order to do this, the DDVA in [12] stops the model checker just before conditional branch points and pointer dereferences are processed and runs a forward analysis. This forward analysis determines a partial path that the program takes in the future and resolves memory aliasing. This forward analysis is terminated either at a user-specified explore depth or at a state with a non-deterministic assignment to a variable. Non-determinism causes states to have uncertain futures thus future information cannot be used to determine dead variables before a point of non-determinism. Having a partial path through the CFG allows the analysis to use program locations and variable valuations to more precisely 
determine dead variable sets. The algorithm prunes off portions of the program that are now known to be unreachable given the observed program locations and variable valuations. The normal SDVA is then run on this reduced program to find more precise sets of dead variables.

Although the DDVA in [12] may find more precise sets of dead variables than SDVA, it presents two issues. The first issue is that there is no correlation between explore depths and state space reductions. This is a consequence of the starting point for each forward analysis and the fact that states cannot have their dead variable sets updated once they have been stored in the Visited set. The algorithm does not run a new forward analysis until the model checker runs past the end of the last forward analysis. Consequently, smaller explore depths have shorter analyses but the analyses happen more often. Whereas bigger explore depths have longer analyses, but the analyses are less frequent. An example of such a situation is illustrated in Figure 1.1.

Figure 1.1 demonstrates how longer explore depths do not always translate to greater state space reductions. In the figure, each box on the left represents a state in the search stack in the model checker. Of particular note are states 10 and 18, where $a$ is defined and then redefined such that $a$ is dead from state 10 to state 17 . In the DDVA of [12], the forward analysis needs to reach state 18 to discover that $a$ is dead. The way the algorithm is designed, it can only declare $a$ dead in the window of states generated after the start of the forward analysis and before the next nondeterministic assignment. In the forward analysis patterns on the right, each empty rectangle represents the window of states explored by a single forward analysis. The analysis pattern with the smaller explore depth finds that $a$ is dead on the second analysis, and since the analysis starts at state 10, can declare $a$ dead in states 10 through 17 . The pattern with the bigger explore depth also finds that $a$ is dead on its second analysis, but since the analysis starts at state 17 , it can only declare $a$ dead 


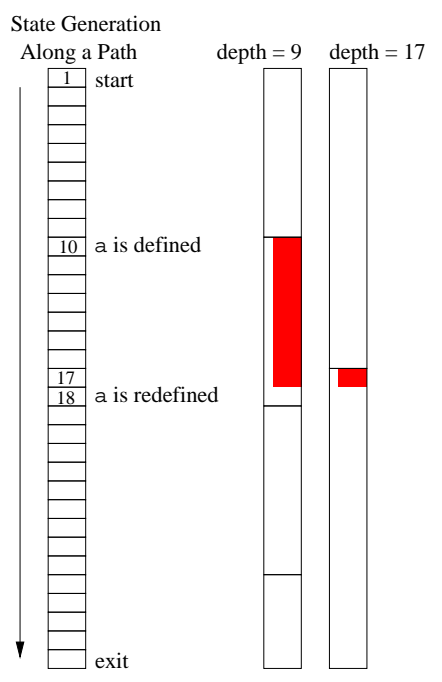

Figure 1.1: On the left is the search stack with the variable $a$ defined at state 10 and then redefined at state 18. On the right are two patterns of forward analyses with different explore depths. Highlighted regions show where each analysis marks $a$ as dead.

at state 17; thus, it is impossible to know a priori the explore depth to produce the best state space reduction without further analysis of the program structure.

The second issue with the DDVA in [12] is that the true dead variable set for a state is not discovered no matter what explore depth is used. Once states are generated and stored in the Visited set they cannot have their dead variable sets updated even if more dead variables are discovered. Any new information about dead variables discovered in a dynamic dead variable analysis is only applied to a small window of states that begins at the start of a forward analysis and ends at a point of non-determinism or the explore depth. This limitation is due to the data structure used to store states and prevents the DDVA algorithm in [12] from achieving the maximal state space reduction even when the best explore depth is used. The goal of this work is to formally define the maximal reduction from DDVA and present an on-the-fly algorithm for computing it. 


\section{Chapter 2}

\section{DVA Maximal Reduction}

The dead variable abstraction in this work relies on the states and execution paths in the reachable state space and the control flow graph (CFG) of the system being verified. A state $s$ is a mapping of variables to a finite domain or $\top, s: V \longrightarrow$ $D \cup\{\top\}$, where $V$ is the set of all variables in the system, $D$ is a finite domain, and $\top$ represents an unconstrained or abstracted variable. We use the symbol $S$ to represent the set of all possible mappings of variables to the domain or $T$. For simplicity, we assume a single initial state, denoted by $s_{0}$, that contains the initial mapping; although, the results readily extend to systems with multiple initial states.

A control flow graph is a tuple, $(N, E)$, where $N$ is a set of nodes and $E \subseteq$ $N \times N$ is a set of edges connecting nodes. Each node $\alpha$ in the CFG represents a transition that executes atomically in the system. A transition $\alpha \subseteq S \times S$ relates a state with its next state. A transition is enabled in $s$ if and only if there exists an $s^{\prime}$ such that $\alpha\left(s, s^{\prime}\right)$ holds. A transition is deterministic if and only if for every state $s$ there is at most one $s^{\prime}$ such that $\alpha\left(s, s^{\prime}\right)$ holds. The CFG is used in an iterative dataflow analysis to find dead variables [13]. SDVA and the DDVA in [12] use a CFG to find dead variables in the program. This work uses execution paths for the analysis.

An execution path, $\pi=s_{0} \stackrel{\alpha_{0}}{\rightarrow} s_{1} \stackrel{\alpha_{1}}{\rightarrow} \cdots$, is a finite or infinite sequence of states and transitions such that $s_{0}$ is the initial state and for every $i, \alpha_{i}\left(s_{i}, s_{i+1}\right)$ is a valid transition and $\left(\alpha_{i}, \alpha_{i+1}\right) \in E$ is a valid edge in the CFG. A path suffix $\pi^{i}$ is the suffix of the execution trace $\pi$ starting at $s_{i}$. The set of all states that are in traces that 
begin with $s_{0}$ and contain only the transitions in $E$ constitute the reachable state space of the system $S_{R}$.

The formal definition we use to mark live and dead variables in a trace makes use of some basic predicates. The predicate $\operatorname{de} f(v, \alpha)$ is true when the variable $v$ is defined by the transition $\alpha$. Similarly, $\operatorname{used}(v, \alpha)$ is true when $v$ is used by $\alpha$. We now give the definition of a variable being live in a state of an execution path:

Definition 1 A variable $v$ is live in a transition $\alpha_{i}$ of an execution path $\pi^{i}=s_{i} \stackrel{\alpha_{i}}{\rightarrow}$ $s_{i+1} \stackrel{\alpha_{i+1}}{\rightarrow} \cdots$ if and only if:

- there exists a $j \geq i$ such that used $\left(v, \alpha_{j}\right)$ and

$$
\text { - } \neg \text { def }\left(v, \alpha_{k}\right) \text { for all } i<k<j
$$

We use this definition of live variables in the function $\operatorname{live}\left(\pi^{i}, v\right)$, which takes $\pi^{i}$, the suffix of the execution trace $\pi$ starting at $s_{i}$, and returns whether the variable $v$ is live in the first state on the trace. If a variable is not live in a state then it is dead. Intuitively, a dead variable is a variable whose current valuation is not used on any future path.

Variables mapped to $\top$ are abstracted and unconstrained. In this way, a state that has abstracted variables can represent many different states. The set of all abstracted variables in a state $s$ is $\operatorname{abstract}(s)=\{v \mid s(v)=\top\}$ and the set of concrete variables is concrete $(s)=\{v \mid s(v) \in D\}$.

In order to compare and match states that have differing sets of abstracted variables we define a relation between two states called contains denoted $\preceq_{c}$.

Definition 2 A state $s^{\prime}$ is contained in $s$, denoted $s^{\prime} \preceq_{c} s$ if:

- $\operatorname{abstract}\left(s^{\prime}\right) \subseteq \operatorname{abstract}(s)$ and

- For all variables $v$ in concrete $\left(s^{\prime}\right), s^{\prime}(v)=s(v)$ 
A state is contained in another state if the set of dead variables of the first state are a subset or equal to the set of dead variables of the second state and variables that are live in both states are equal.

SDVA only uses the information available in the CFG of the program to do the analysis. Because of this, SDVA admits infeasible paths and produces an imprecise set of dead variables. Dead variable analysis is more precise if it uses data available during run-time, such as variable valuations and next state information, in addition to information in the CFG to determine exactly which path through the CFG is feasible in an execution trace and then only use this path to find dead variables. When a precise execution path through the CFG is used to find dead variables, the true dead variable sets for every state on the trace can be calculated. Finding the true dead variable set for each state in the reachable state space produces an abstract state space that is a DVA maximal reduction of the concrete state space.

Definition 3 An abstract state space $S_{R}^{\prime}$ is a DVA maximal reduction of the concrete state space $S_{R}$ if and only if:

- For every reachable execution trace starting at the initial state $\pi=s_{0} \stackrel{\alpha_{0}}{\rightarrow} s_{1} \stackrel{\alpha_{1}}{\rightarrow}$ .. in the concrete state space, there exists an abstract execution trace $\pi^{\prime}=$ $s_{0}^{\prime} \stackrel{\alpha_{0}}{\rightarrow} s_{1}^{\prime} \stackrel{\alpha_{1}}{\rightarrow} \cdots$ such that for all $i, s_{i} \preceq_{c} s_{i}^{\prime}$ and $s_{i}^{\prime} \in S_{R}^{\prime}$

- For all states $s^{\prime}$ in $S_{R}^{\prime}$, and for all variables $v$ in $V$, if the value of $v$ in $s^{\prime}$ is not $\top$, then there exists a reachable concrete trace $\pi=s_{0} \stackrel{\alpha_{0}}{\rightarrow} s_{1} \stackrel{\alpha_{1}}{\rightarrow} \cdots$ and an $i \geq 0$ such that $s_{i} \preceq_{c} s^{\prime}$ and live $\left(\pi^{i}, v\right)$

The original DDVA in [12] uses some runtime information to refine SDVA and find more dead variables; however, it is not able to construct a DVA maximal reduction of a concrete state space and occasionally creates an abstract state space that is no smaller than the state space produced using SDVA. The dynamic dead variable analysis in this work implements Definition 4 on-the-fly to produce a DVA maximally 
reduced state space in the absence of non-determinism. In the presence of nondeterminism, our dynamic dead variable analysis produces the closest approximation to a DVA maximally reduced state space that is possible to produce on-the-fly.

\subsection{Maximal Dynamic Dead Variable Analysis}

Our DDVA algorithm achieves a DVA maximal reduction by analyzing fully determined execution paths through the program instead of partial future paths generated from a forward analysis. Our algorithm is not maximal when non-determinism is present in the program being verified because non-determinism makes the future paths uncertain. A greater treatment of the effect of non-determinism can be found at the end of this section. A fully determined execution path is a single execution path that has been fully explored; it generates no more unique states. An execution path that has reached the exit of the program or a path that has reached an already visited state (representing a path that has entered an infinite loop or merged into an already explored path) are the two kinds of fully determined execution paths. Whenever the search generates a fully determined path, a dead variable analysis is performed. The equation in Definition 2 is used to calculate the new sets of dead variables for each state in the path starting with the last state in the path. The exception to this is when a prefix for a trace is unique but all states in its suffix are already in the Visited set. In this case, we can use the dead variable information we already calculated for the suffix to start calculating the dead variables at the last state of the prefix.

When the model checker fully resolves an execution path through the program, the dead variable analysis may find more dead variables for states that have already been explored. A full execution path reveals dynamic run time information of all of the states in the path, allowing the analysis to positively declare variables live or dead. Updating the dead variable sets of visited states requires that they be re-stored in the Visited set. In order to avoid storing states that are later found to be duplicates 
when their dead variable sets are updated, we use the contains relation to ascertain whether a state is unique even before its final dead variable set is generated. In our algorithm, if $s^{\prime} \preceq_{c} s, s^{\prime}$ is a newly generated state, and $s \in$ Visited, then $s^{\prime}$ is not inserted into Visited, because it is contained in $s$. We do not need to add $s^{\prime}$ to the Visited set because once its true dead variable set is discovered, it is a duplicate of $s$. This pre-emptive duplicate detection saves us from having to generate and store states that are later found to be duplicates.

The new algorithm to dynamically find dead variables, shown in Figure 2.1. is remarkably simpler than the work in [12]. The function dfs performs a standard depth first search to enumerate the entire state space of the model. Stack is the depth first search stack. An entry in Stack consists of $(s, A)$, with $s$ being a state that includes the location and $A$ being a set of transitions that can be applied to the state to get a next state and location. For our Visited set, we use a hash table that implements the contains relation to compare states. The function $a(s)$ takes a state $s$ and returns the set $A, a: s \longrightarrow A$. A transition $\alpha \in A$ maps a current state onto a next state as defined previously. When a duplicate state is generated (line 11) or the exit is reached (line 16), model checking is suspended and a dead variable analysis is run. In the case that the exit of the program is reached, updateDeadVars is called with null because the entire trace is contained in Stack. When a duplicate state is reached, updateDeadVars is called with the state in the hash table that matched the newly generated state.

The equation used in updateDeadVars to calculate dead variables sets in a state requires as input the previous state's set of dead variables. The variable used for this, PreviousDeadVars, is initialized to all variables at line $\mathbf{2 2}$ when the exit is reached due to the fact that all variables are dead at the exit. When a partial path is in Stack and a path suffix is in Visited, we initialize PreviousDeadVars to be the dead variables in the state we matched on, line $\mathbf{2 4}$. Finally, in cases where the exit 
is not reached because the modeled program enters an infinite loop, the analysis is started with PreviousDeadVars being empty, line 26, as we cannot determine what the previous state's dead variable set is without entering into an infinite loop ourselves. When a state maps to a non-deterministic assignment in the program, as indicated by the return value of nonDeterminism( strace $)$, PreviousDeadVars is set to the empty set, because dead variables discovered after a point of non-determinism cannot be used to calculate the set of dead variables for states before the non-determinism. This point is explained in greater depth at the end of this section.

The equation for the definition of a dead variable is applied at line $\mathbf{3 1}$ of updateDeadVars to find the set of dead variables for each state in the trace. The function $\operatorname{def}(A)=\{v \mid \forall \alpha \in A, \operatorname{def}(\alpha, v)\}$ returns the set of variables that are defined in a set of transitions and the function $\operatorname{used}(A)=\{v \mid \forall \alpha \in A$, used $(\alpha, v)\}$ returns the set of variables that are used by a set of transitions. If the analysis finds more dead variables than are currently in the state, the states in Stack are updated with their new dead variable sets. Variables that are always live, such as the location, are never abstracted. The updated states are re-stored in the hash table using the function replace (Visited, $s_{\text {trace }}, s^{\prime}$ ) (line $\mathbf{3 4}$ ).

The following is an example run of the algorithm in Figure 2.1 that produces the state space shown in Figure 2(b). Since some states shown in Figure 2(a) are produced and then later have their dead variable sets updated to become the states in Figure 2(b), we add a superscript $a$ or $b$ to states that differ between the two figures. Our model checking run starts with $s_{0}$ as our start state. The state $s_{0}$ is pushed onto Stack at line $\mathbf{2}$ and then the depth-first search is called at line $\mathbf{3}$. In the main loop of $\mathbf{d f s}, s_{0}$ is retrieved from Stack. Line 8 chooses a transition $\alpha$ from $s_{0}$ 's transition set, if there is more than one transition, and removes the transition from the set. Then line $\mathbf{9}$ uses the transition to produce $s_{1}$ from program location $\mathbf{1}$ of Figure 1. We check for uniqueness of the newly generated state in lines $\mathbf{1 0}$ and $\mathbf{1 1 .}$ 
If the state is not contained in any other state in Visited, then it is a unique state. The new state in this example is unique so we add it to Vistited and then push it onto Stack at lines $\mathbf{1 4}$ and $\mathbf{1 5}$. We need to perform a dead variable analysis on each trace after it has been generated, so we check if this trace has finished at line $\mathbf{1 6}$ by checking to see if the current state's location is the program exit.

The current trace has not reached the exit so we return to the top of the loop and take $s_{1}$ off of the top of Stack. The state $s_{1}$ has a single action in its action set. This action is used to produce $s_{5}$ which is added to Visited and Stack. The third time through the main loop of $\mathbf{d f s}, s_{5}$ is retrieved from Stack at line $\mathbf{6}$. The state $s_{8}$, the successor of $s_{5}$, is generated and pushed onto Stack. Since $s_{8}$ is generated at the exit location, we call updateDeadVars at line 17. All variables are dead at the end of the program so the set PreviousDeadVars is set to contain all the variables in the program at line $\mathbf{2 2}$. We iterate backwards through the trace calculating the dead variables for each state starting at the last state. The dead variables of the current state are calculated using the formula on line $\mathbf{3 1}$ and then the appropriate variables are marked as dead at line 32. In this example, no new dead variables are found, so we return from updateDeadVars. The third time through the main $\mathbf{d f s}$ loop, $s_{8}$ is at the top of Stack. It does not require a dead variable analysis, and it has an empty action set, so it is left off of Stack, and we look at $s_{5}$. The state $s_{5}$ also has no more children, so it is also popped off Stack and then the same process occurs for $s_{1}$.

The next action in $s_{0}$ 's action set produces $s_{3}^{a}$. The state $s_{3}^{a}$ does not trigger a dead variable analysis and the successor of $s_{3}^{a}, s_{6}^{a}$, also does not trigger an analysis. The next state, $s_{9}$, is at the exit, so another dead variable analysis is run. This time the variable $b$ is found to be dead at program locations $\mathbf{2}$ and $\mathbf{3}$. Marking $b$ as dead in $s_{3}^{a}$ and $s_{6}^{a}$ produces the states $s_{3}^{b}$ and $s_{6}^{b}$ which replace the previous states at lines 33 and 34.

After returning from updateDeadVars, $s_{9}, s_{6}$, and $s_{3}$ are popped off of Stack. 
The next successor of $s_{0}$ is $s_{4}$ which is contained in $s_{3}^{b}$, so it is not added to the Stack or Visited. Only $s_{0}$ is in Stack when updateDeadVars is called so no new dead variables are found. Now that $s_{0}$ 's action set is empty, it is popped from Stack and state generation has completed.

Our DDVA algorithm is designed on the definitions in the previous section. As such, we claim that using our algorithm to model check single procedure programs without non-determinism produces DVA maximally reduced state spaces on-the-fly by implementing Definitions 2 - 4 . However, the presence of non-deterministic assignments to variables can affect the future path from a state so that a state with a non-deterministic assignment can have more than one possible future. These multiple futures of a single state may cause the state to have different sets of dead variables. It is possible that the non-determinism does not actually affect the state's dead variable set, but the only way to know for sure is to examine the entire reachable state space; however, once the entire reachable state space is produced, model checking has finished and there is no longer a need to find more dead variables.

An example of how an execution path can affect states produced before the point of non-determinism is presented in Figure 2.2. The function get_input represents non-deterministic input from an outside source that ranges over a large finite domain. The variable $a$ is dead at location 2 if $c$ is greater than 2 and the path goes through location 4 . A state generated at location $\mathbf{2}$ could not have $a$ marked as dead because $c$ might be assigned a value less than or equal to 2, making $a$ live. It is possible that every single value returned by get_input at location 2 during model checking is greater than 2, which means we can mark $a$ dead at location 2 ; however, the only way to check if get_input always returns a value greater than 2 is to finish generating the entire reachable state space. Once the entire state space is produced, it is no longer longer necessary to find new dead variables, because model checking is finished. 


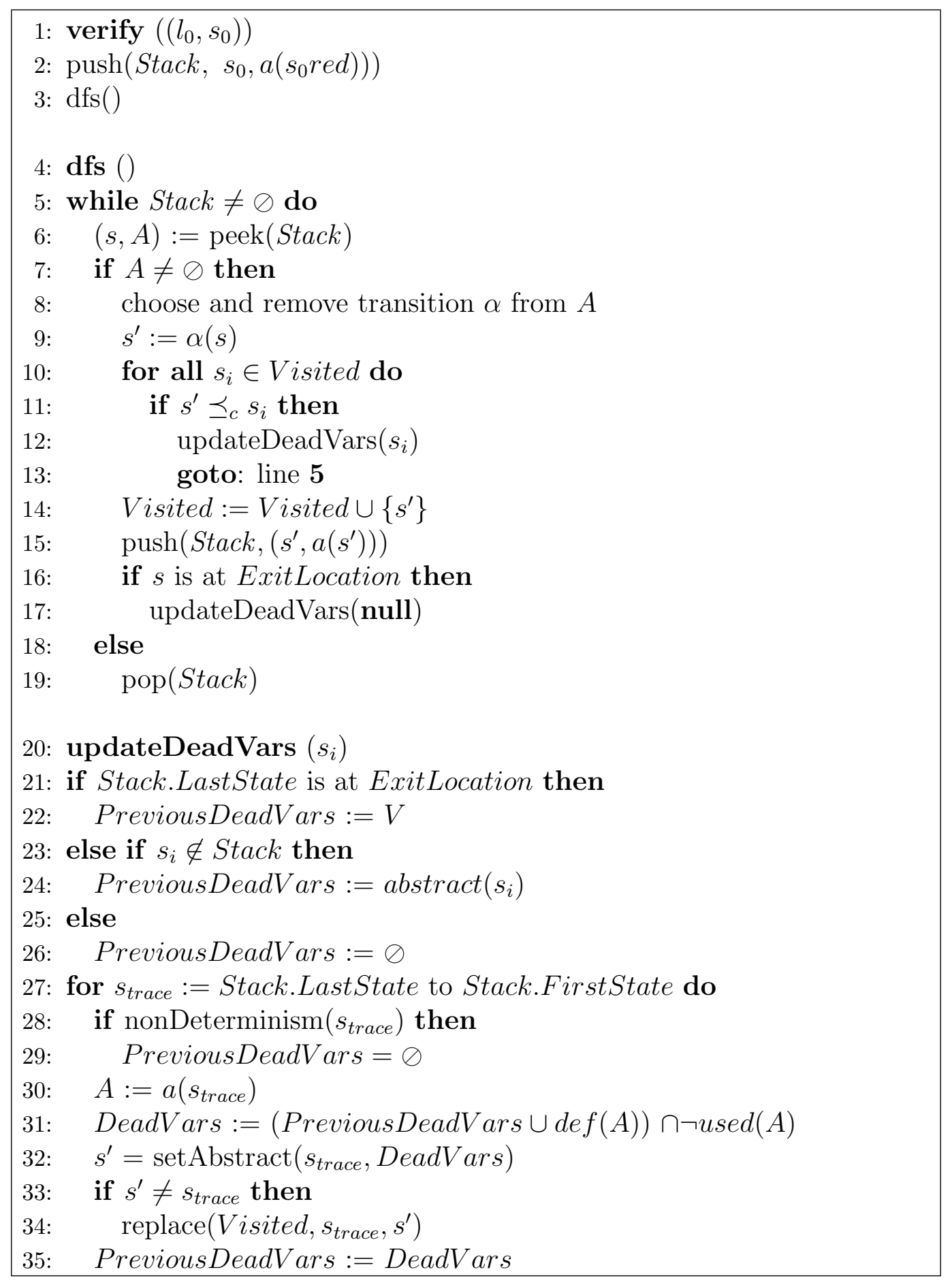

Figure 2.1: Pseudocode of the new DDVA algorithm. 


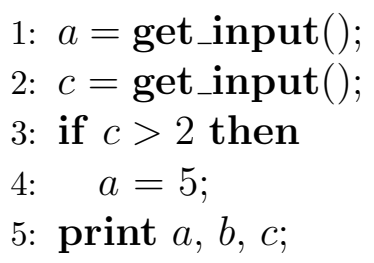

Figure 2.2: A program fragment that has a point of non-determinism that affects what can be declared dead above it.

In order to not incorrectly mark variables as dead in the presence of nondeterminism, dead variable knowledge gained after a non-deterministic assignment cannot be used on states generated before the assignment unless we first generate every possible assignment and future path for the analysis. It is possible that on some models this strategy does find the DVA maximal reduction as it may be the case that the non-determinism in a particular model does not affect dead variable sets in preceding states. We cannot determine on-the-fly whether this is the case, so our algorithm produces state spaces that are not technically DVA maximally reduced when non-determinism is present; however, for on-the-fly model checking without non-determinism, our algorithm produces the maximal possible state space reduction from dead variable analysis.

\section{$2.2 \quad$ Results}

We implemented our DDVA algorithm in the Estes model checker developed at the BYU Software Model Checking Lab [14]. Estes uses the GNU debugger as a state generator in order to verify software at the object code level. Since a single line of code from a high level language can easily translate into 2 or more object code instructions, ways to reduce the size of the explored state space are invaluable. The specific simulator we use as our state generator is based on the Motorola 68hc11

processor and can be found in the Gnu Debugger (GDB) [15]. We use the tools found in the GEL collection of libraries [16] to compile $\mathrm{C}$ source code into the binary files 
that run in the simulator.

In order to implement the contains relation, we need to be able to compare new states with existing states to see if the new state is contained in another state; however, comparing each new state with all the existing states in the Visited set is too unwieldy as the set becomes larger. In order to mitigate this problem, we use a chained hash table, where each chain has a subset of variables that are all equal and that can never be dead. Since we need to compare states to other states that may have a larger set of dead variables, we only use variables that are never dead to hash into the correct chain. In all of our examples, we mark the registers and location as the set of variables that are never dead and hash on this set to find the correct chain. Once the correct chain is found, the state is compared to each of the states in the chain until an exact match or containing state is found, or the end of the chain is reached. If a match or containing state is found, then the new state is discarded. If the new state is unique, it is simply appended to the end of the chain.

We compare the implementation of our DDVA algorithm against normal model checking, model checking with SDVA, and the best and worst runs of the DDVA in [12]. We compare the different techniques running on 6 different models in the following areas:

- States generated: Size of the Visited set at the end of model checking.

- Wall clock time: Total time taken to finish model checking. This includes the time used by the operating system and other services running on the computer.

- Total memory used: The total amount of memory used by the model checker to complete a model checking run.

- Abstraction time: Total amount of time taken in the dead variable analyses.

We test the algorithms on a number of artificial and real world tests including the main test used to benchmark the DDVA in [12]. The first three models are artificial with 
no real world objective other than to showcase the kind of state space reductions that are possible with a dynamic dead variable analysis. The last three models are mockups of real world functions or programs than can be found in embedded platforms or general purpose computers. The results are shown in Figure 2.3 and Figure 2.4.

The data in Figure 2.3 and Figure 2.4 show how the DDVA in [12] either results in no better reduction than SDVA or has widely varying results depending on the explore depth. Our DDVA on the other hand always has a smaller state space than SDVA, and thus, always has lower memory usage than all of the other methods. For smaller models, the reduced state count results in faster run times; however, on larger models, the chains in the hash table become very long and cause the algorithm to take longer than the other methods. For simplicity, the DDVA algorithm in [12] is referred to as original in the tables, while our algorithm is referred to as maximal.

The easy3 model is a program with several global integer variables that nondeterministically receive a value at the beginning of the program. The rest of the program contains conditional branches and, depending on values of the variables, all but one variable becomes dead in each branch. The results are shown in the top table of Figure 2.3. This is an example that benefits greatly from dead variable analysis. The original DDVA discovers dead variables at the exact same point that SDVA finds dead variables in this example and incurs the time penalty of extra analyses for no state space reduction. Our DDVA reduces the state space and is only slightly slower than SDVA. The original DDVA performs more analyses and thus takes almost twice as long as our DDVA to do its abstraction and yet gains nothing over the static analysis. Our DDVA produces a 35\% smaller state space and correspondingly has a lower peak memory usage.

The littleBranch model is similar to easy3 although it contains nested conditionals which the original DDVA can take advantage of with the right explore depth. The results are shown in the middle table of Figure 2.3. This model, however small, 
Model Name: easy3, Lines of Code: 38

\begin{tabular}{|c|c|c|c|c|c|}
\hline Analysis & $\begin{array}{c}\text { Explore } \\
\text { Depth }\end{array}$ & $\begin{array}{c}\text { States } \\
\text { Generated }\end{array}$ & $\begin{array}{c}\text { Total } \\
\text { Time }\end{array}$ & $\begin{array}{c}\text { Memory } \\
\text { Used (MB) }\end{array}$ & $\begin{array}{c}\text { Abstraction } \\
\text { Time }\end{array}$ \\
\hline None & N/A & 34640 & $0 \mathrm{~m} 12.764 \mathrm{~s}$ & 34.5 & $0.0 \mathrm{~s}$ \\
\hline Static & N/A & 15814 & $0 \mathrm{~m} 6.605 \mathrm{~s}$ & 33.80 & $0.001 \mathrm{~s}$ \\
\hline Original best & 2 & 15814 & $0 \mathrm{~m} 10.765 \mathrm{~s}$ & 34.46 & $3.792 \mathrm{~s}$ \\
\hline Original worst & 2 & 15814 & $0 \mathrm{~m} 10.765 \mathrm{~s}$ & 34.46 & $3.792 \mathrm{~s}$ \\
\hline Maximal & N/A & 10330 & $0 \mathrm{~m} 8.105 \mathrm{~s}$ & 25.5312 & $2.017 \mathrm{~s}$ \\
\hline
\end{tabular}

Model Name: littleBranch, Lines of Code: 57

\begin{tabular}{|c|c|c|c|c|c|}
\hline Analysis & $\begin{array}{c}\text { Explore } \\
\text { Depth }\end{array}$ & $\begin{array}{c}\text { States } \\
\text { Generated }\end{array}$ & $\begin{array}{c}\text { Total } \\
\text { Time }\end{array}$ & $\begin{array}{c}\text { Memory } \\
\text { Used (MB) }\end{array}$ & $\begin{array}{c}\text { Abstraction } \\
\text { Time }\end{array}$ \\
\hline None & N/A & 864 & $0 \mathrm{~m} 0.442 \mathrm{~s}$ & 30.9 & $0.0 \mathrm{~s}$ \\
\hline Static & N/A & 721 & $0 \mathrm{~m} 0.405 \mathrm{~s}$ & 31.4 & $0.001 \mathrm{~s}$ \\
\hline Original best & 6 & 658 & $0 \mathrm{~m} 0.344 \mathrm{~s}$ & 31.43 & $0.074 \mathrm{~s}$ \\
\hline Original worst & 2 & 721 & $0 \mathrm{~m} 0.34 \mathrm{~s}$ & 31.43 & $0.0492 \mathrm{~s}$ \\
\hline Maximal & N/A & 530 & $0 \mathrm{~m} 0.223 \mathrm{~s}$ & 23.79 & $0.0138 \mathrm{~s}$ \\
\hline
\end{tabular}

Model Name: multiBranch, Lines of Code: 140

\begin{tabular}{|c|c|c|c|c|c|}
\hline Analysis & $\begin{array}{c}\text { Explore } \\
\text { Depth }\end{array}$ & $\begin{array}{c}\text { States } \\
\text { Generated }\end{array}$ & $\begin{array}{c}\text { Total } \\
\text { Time }\end{array}$ & $\begin{array}{c}\text { Memory } \\
\text { Used (MB) }\end{array}$ & $\begin{array}{c}\text { Abstraction } \\
\text { Time }\end{array}$ \\
\hline None & N/A & 294515 & $1 \mathrm{~m} 49.170 \mathrm{~s}$ & 87.1 & N/A \\
\hline Static & N/A & 217454 & $1 \mathrm{~m} 21.780 \mathrm{~s}$ & 74.87 & $0.002 \mathrm{~s}$ \\
\hline Original best & 16 & 176651 & $1 \mathrm{~m} 41.458 \mathrm{~s}$ & 75.79 & $42.67 \mathrm{~s}$ \\
\hline Original worst & 5 & 217478 & $2 \mathrm{~m} 10.965 \mathrm{~s}$ & 83.46 & $46.35 \mathrm{~s}$ \\
\hline Maximal & N/A & 145440 & $2 \mathrm{~m} 36.640 \mathrm{~s}$ & 57.99 & $7.513 \mathrm{~s}$ \\
\hline
\end{tabular}

Figure 2.3: Results for 3 artificial models. All 3 models are disgined to showcase the benefits of using DDVA.

illustrates the difficulty in achieving a good result with the original DDVA. Our DDVA, on the other hand, gives the largest state space reduction, takes the least time to complete, and is able to do this every time without a user specified depth bound.

The multiBranch model shown in Figure 2.3 is a much larger version of the littleBranch model that is used to test the original DDVA. In addition to having deeper nesting than littleBranch, multiBranch makes use of local variables that are referenced as an offset from the frame pointer. Whenever there is an upcoming pointer 
dereference, SDVA is forced to declare all variables live. The results from this model are shown in the lower table of Figure 2.3. This is a good example of a situation where DDVA is engineered to surpass the performance of SDVA; however, again the performance of the original DDVA is unpredictable, and at its worst, generates more states than the static analysis due to the strict state comparison in the hash table. Please note that although our DDVA generates the smallest state space in this example it incurs a higher run time due to the long chains in the chained table.

Figure 2.4 gives the results from the lexer, robot and bintree models. The lexer model is patterned after a function in a simple lexical analyzer. The model simulates input as a string of characters which the function reads and then returns a token based on what is in the first one or two characters. The robot model simulates a line following robot with three sensors. The robot changes the speed of its left and right motors based on input from the three sensors. In both of these models, our DDVA has the smallest state space and lowest memory usage while taking equal or less time to complete. The bintree model is the only model with a loop in it. This model searches a binary tree for a specific node. Due to algorithmic limitations, the original DDVA typically does not perform well on models with loops. Our DDVA does much better because it analyzes entire traces through the program which is equivalent to unrolling the loop as many times as needed and then performing dead variable analysis on the unrolled loop as shown in the bottom table of Figure 2.4 . 
Model Name: lexer, Lines of Code: 92

\begin{tabular}{|c|c|c|c|c|c|}
\hline Analysis & $\begin{array}{c}\text { Explore } \\
\text { Depth }\end{array}$ & $\begin{array}{c}\text { States } \\
\text { Generated }\end{array}$ & $\begin{array}{c}\text { Total } \\
\text { Time }\end{array}$ & $\begin{array}{c}\text { Memory } \\
\text { Used (MB) }\end{array}$ & $\begin{array}{c}\text { Abstraction } \\
\text { Time }\end{array}$ \\
\hline None & N/A & 262843 & $1 \mathrm{~m} 28.391 \mathrm{~s}$ & 66.9 & $0.0 \mathrm{~s}$ \\
\hline Static & N/A & 226169 & $1 \mathrm{~m} 17.633 \mathrm{~s}$ & 66.32 & $0.002 \mathrm{~s}$ \\
\hline Original best & 2 & 225370 & $1 \mathrm{~m} 51.479 \mathrm{~s}$ & 71.30 & $31.66 \mathrm{~s}$ \\
\hline Original worst & 3 & 226172 & $1 \mathrm{~m} 53.866 \mathrm{~s}$ & 71.13 & $33.46 \mathrm{~s}$ \\
\hline Maximal & N/A & 74024 & $1 \mathrm{~m} 45.56 \mathrm{~s}$ & 37.69 & $4.898 \mathrm{~s}$ \\
\hline
\end{tabular}

Model Name: robot, Lines of Code: 55

\begin{tabular}{|c|c|c|c|c|c|}
\hline Analysis & $\begin{array}{c}\text { Explore } \\
\text { Depth }\end{array}$ & $\begin{array}{c}\text { States } \\
\text { Generated }\end{array}$ & $\begin{array}{c}\text { Total } \\
\text { Time }\end{array}$ & $\begin{array}{c}\text { Memory } \\
\text { Used (MB) }\end{array}$ & $\begin{array}{c}\text { Abstraction } \\
\text { Time }\end{array}$ \\
\hline None & N/A & 35865 & $0 \mathrm{~m} 12.838 \mathrm{~s}$ & 35.3 & $0.0 \mathrm{~s}$ \\
\hline Static & N/A & 27940 & $0 \mathrm{~m} 10.377 \mathrm{~s}$ & 35.6 & $0.002 \mathrm{~s}$ \\
\hline Original best & 2 & 27940 & $0 \mathrm{~m} 18.675 \mathrm{~s}$ & 36.21 & $7.947 \mathrm{~s}$ \\
\hline Original worst & 2 & 27940 & $0 \mathrm{~m} 18.675 \mathrm{~s}$ & 36.21 & $7.947 \mathrm{~s}$ \\
\hline Maximal & N/A & 27784 & $0 \mathrm{~m} 11.494 \mathrm{~s}$ & 29.21 & $0.552 \mathrm{~s}$ \\
\hline
\end{tabular}

Model Name: bintree, Lines of Code: 31

\begin{tabular}{|c|c|c|c|c|c|}
\hline Analysis & $\begin{array}{c}\text { Explore } \\
\text { Depth }\end{array}$ & $\begin{array}{c}\text { States } \\
\text { Generated }\end{array}$ & $\begin{array}{c}\text { Total } \\
\text { Time }\end{array}$ & $\begin{array}{c}\text { Memory } \\
\text { Used (MB) }\end{array}$ & $\begin{array}{c}\text { Abstraction } \\
\text { Time }\end{array}$ \\
\hline None & N/A & 157828 & $1 \mathrm{~m} 0.608 \mathrm{~s}$ & 66.5 & $0.0 \mathrm{~s}$ \\
\hline Static & N/A & 154084 & $1 \mathrm{~m} 1.061 \mathrm{~s}$ & 68.4 & $0.005 \mathrm{~s}$ \\
\hline Original best & 6 & 150964 & $2 \mathrm{~m} 14.807 \mathrm{~s}$ & 73.74 & $72.09 \mathrm{~s}$ \\
\hline Original worst & 2 & 154084 & $2 \mathrm{~m} 7.356 \mathrm{~s}$ & 71.47 & $64.87 \mathrm{~s}$ \\
\hline Maximal & N/A & 103839 & $1 \mathrm{~m} 7.530 \mathrm{~s}$ & 52.62 & $16.34 \mathrm{~s}$ \\
\hline
\end{tabular}

Figure 2.4: Results for 3 real-world models. The lexer model is a simple lexical analyzer. The robot model simulates a line following robot. The bintree model searches a binary tree for a specific node. 


\section{Chapter 3}

\section{Conclusions and Future Work}

Dead variable analysis is an effective means of reducing the size of the explored state space in model checking while retaining all relevant behaviors of the system. Dynamic dead variable analysis provides a way of finding a larger set of dead variables for each state resulting in even smaller state spaces than those generated using SDVA. Our DDVA greatly improves upon the ideas set forth in the original DDVA of [12] by eliminating the dependence on a user specified explore depth and by producing a DVA maximally reduced state space for models with no non-determinism and the closest possible approximation to a DVA maximally reduced state space in models that contain non-determinism. This results in state spaces that, at worst, are no larger than those generated by SDVA, and are often much smaller.

Our maximal DDVA algorithm is currently limited to single procedure programs. Future work focuses on modifying our DDVA algorithm to work on multiprocedural programs. The easiest way to do this is to declare all global variables as live, and treat every procedure and its local variables as a separate program. As the program returns from a procedure, a dynamic dead variable analysis is run on the trace of states generated through the procedure and dead variables sets for states generated in the procedure are updated.

Other areas of future work include finding ways to speed up run time, adapting the algorithm to different searches, and using a more efficient way of representing dead variables. The current implementation of the algorithm suffers from an increase in run 
time on large models that can make state space exploration infeasible. This increase in run time comes from the use of a chained hash table and the contains relation. An avenue for future work would be to look into ways to mitigate this problem. Another direction for future work adapts DDVA to work with other search algorithms such as breadth-first search. The benefit of breadth-first search is that paths that reach an error state are guaranteed to be the shortest path to the error. Lastly, the current data structure used to mark dead variables is highly inefficient. Some future work could be dedicated to creating data structures that take less memory to store dead variable information. 


\section{Bibliography}

[1] G. J. Holzmann, The Spin Model Checker: Primer and Reference Manual. Addison-Wesley, 2003.

[2] A. Cimatti, E. M. Clarke, F. Giunchiglia, and M. Roveri, "NUSMV: A new symbolic model verifier," in Computer Aided Verification, 1999, pp. 495-499. [Online]. Available: citeseer.ist.psu.edu/cimatti99nusmv.html

[3] M. Robby and J. Dwyer, "Bogor: an extensible and highly-modular software model checking framework," 2003. [Online]. Available: citeseer.ist.psu.edu/ dwyer03bogor.html

[4] T. A. Henzinger, R. Jhala, R. Majumdar, , and G. Sutre, "Software verification with Blast," in Proceedings of the 10th International Workshop on Model Checking of Software (SPIN), ser. Lecture Notes in Computer Science, T. Ball and S. Rajamani, Eds., vol. 2648, Portland, OR, May 2003, pp. 235-239.

[5] K. Havelund and T. Pressburger, "Model checking java programs using java pathfinder," 1998. [Online]. Available: citeseer.ist.psu.edu/havelund98model. html

[6] E. M. Clarke, O. Grumberg, and D. E. Long, "Model checking and abstraction," ACM Trans. on Programming Languages and Systems, vol. 16, no. 5, pp. 15121542, September 1994.

[7] G. J. Holzmann, "The engineering of a model checker: the gnu i-protocol case study revisited," in Proc. of the 6th Spin Workshop, ser. Lecture Notes in Computer Science, vol. 1680. Toulouse, France: Springer Verlag, 1999. [Online]. Available: citeseer.ist.psu.edu/holzmann99engineering.html

[8] Y. Dong and C. R. Ramakrishnan, "An optimizing compiler for efficient model checking," in FORTE XII / PSTV XIX '99: Proceedings of the IFIP TC6 WG6.1 Joint International Conference on Formal Description Techniques for Distributed Systems and Communication Protocols (FORTE XII) and Protocol Specification, Testing and Verification (PSTV XIX). Kluwer, B.V., 1999, pp. 241-256. 
[9] J. C. Corbett, M. B. Dwyer, J. Hatcliff, S. Laubach, C. S. Păsăreanu, R. Zheng, and H. Zheng, "Bandera: extracting finite-state models from Java source code," in International Conference on Software Engineering, 2000, pp. 439-448. [Online]. Available: citeseer.nj.nec.com/corbett00bandera.html

[10] M. Bozga, J. Fernandez, and L. Ghirvu, "State space reduction based on live variables analysis," in Static Analysis, 6th International Symposium, SAS '99, Venice, Italy, September 22-24, 1999, Proceedings, ser. Lecture Notes in Computer Science, A. Cortesi and G. Filé, Eds., vol. 1694. Springer, 1999, pp. 164-178.

[11] T. Ball and S. K. Rajamani, "Bebop: A symbolic model checker for boolean programs," in 7th International SPIN Workshop, ser. Lecture Notes in Computer Science, K. Havelund, J. Penix, and W. Visser, Eds., vol. 1885. Springer, August 2000, pp. 113-130. [Online]. Available: citeseer.nj.nec.com/ball00bebop.html

[12] M. S. Lewis and M. D. Jones, "A dead variable analysis for explicit model checking," in ACM SIGPLAN 2006 Workshop on Partial Evaluation and Program, 2006.

[13] A. V. Aho, R. Sethi, and J. D. Ullman, Compilers: principles, techniques, and tools. Boston, MA, USA: Addison-Wesley Longman Publishing Co., Inc., 1986.

[14] E. G. Mercer and M. Jones, "Model checking machine code with the GNU debugger," in 12th International SPIN Workshop, ser. Lecture Notes in Computer Science, vol. 3639. San Francisco, USA: Springer, August 2005, pp. 251-265.

[15] "The gnu project debugger," Available at http://sources.redhat.com/gdb/, 2006.

[16] "Gnu embedded libraries for 68hc11 and 68hc12," Available at http://gel.sourceforge.net/, 2005. 